\title{
Elaboración y caracterización Fisicoquímica de membranas bioplásticas a base de Nopal (Opuntia ficus-indica)
}

\section{Preparation and Physicochemical characterization of bioplastic membranes based on Nopal (Opuntia ficus-indica)}

\author{
GÁLVEZ-CHAN, Rosario Alicia†*, SILVA-ENCINAS, Gabriel Omar y PAREDES-GÁLVEZ, \\ Patricia Alejandra
}

Instituto Tecnológico de Sonora, Departamento de Biotecnología y Ciencias Alimentarias

ID $1^{\mathrm{er}}$ Autor: Rosario Alicia, Gálvez-Chan / ORC ID: 0000-0002-5069-4181, Researcher ID Thomson: S-5902-2018, CVU CONACYT ID: 895013

ID $1{ }^{\text {er }}$ Coautor: Gabriel Omar, Silva-Encinas / ORC ID: 0000-0001-5518-1150, Researcher ID Thomson: S-4845-2018, CVU CONACYT ID: 946843

ID $2^{\text {do }}$ Coautor: Patricia Alejandra, Paredes-Gálvez / ORC ID: 0000-0002-2095-5205, Researcher ID Thomson: S-65222018, CVU CONACYT ID: 946866ID

DOI: 10.35429/JCPE.2019.21.6.40.44

Recibido 09 Agosto, 2019; Aceptado 30 Noviembre, 2019

\section{Resumen}

La presente investigación tiene como objetivo la caracterización fisicoquímica de membranas bioplásticas a base de Nopal (Opuntia ficus-indica) para determinar sus posibles aplicaciones. Las pruebas realizadas para la caracterización fueron: permeabilidad, solubilidad, resistencia, grosor, prueba de gota y análisis químico con el espectrofotómetro de infrarrojo. Hoy en día, se buscan nuevas y mejores alternativas en la obtención de diferentes polímeros que beneficien a la humanidad y al medio ambiente. El reciclado es una opción pero no es una alternativa muy efectiva para todos los plásticos, en cambio, los biopolímeros suponen una solución desde el origen del problema ya que la gran mayoría de ellos su tiempo de biodegradación es mucho menor a la de un plástico común. Los biopolímeros, que en su mayor parte proceden de recursos renovables por lo que se convierten en una interesante alternativa para la industria de los plásticos. Las propiedades físico-químicas de las membranas a base de Nopal (Opuntia ficus-indica), son similares a las membranas de plásticos sintéticos, siendo una alternativa viable para sustituirlos, contribuyendo así, a la sociedad, con una mejor calidad de vida al ser amigable al cuidado del medio ambiente.

Bioplástico, Caracterización, Nopal

\begin{abstract}
The objective of the research is the physicochemical characterization of bioplastic membranes based on Nopal (Opuntia ficus-indica) to determine their possible applications. The tests performed for the characterization were: permeability, solubility, resistance, thickness, drop test and chemical analysis with the infrared spectrophotometer. Today, new and better alternatives are sought in obtaining different polymers that benefit humanity and the environment. Recycling is an option but it is not a very effective alternative for all plastics, however, biopolymers are a solution from the origin of the problem since the vast majority of them their biodegradation time is much less than that of a common plastic. Biopolymers, which for the most part come from renewable resources so they become an interesting alternative for the plastics industry. The physicochemical properties of the Nopal-based membranes (Opuntia ficusindica), are similar to synthetic plastic membranes, being a viable alternative to replace them, thus contributing to society, with a better quality of life being environmentally friendly.
\end{abstract}

Bioplastic, Characterization, Nopal

Citación: GÁLVEZ-CHAN, Rosario Alicia, SILVA-ENCINAS, Gabriel Omar y PAREDES-GÁLVEZ, Patricia Alejandra. Elaboración y caracterización Fisicoquímica de membranas bioplásticas a base de Nopal (Opuntia ficus-indica). Revista de Energía Química y Física. 2019. 6-21: 40-44

\footnotetext{
* Correspondencia al Autor (Correo electrónico: rosario.galvez@itson.edu.mx)

$\dagger$ Investigador contribuyendo como primer Autor
} 


\section{Introducción}

Hoy en día, se buscan nuevas y mejores alternativas en la obtención de diferentes polímeros que beneficien a la humanidad y al medio ambiente. El aumento de los precios internacionales del petróleo, la inestabilidad de la situación geopolítica de las regiones que poseen las grandes reservas mundiales y el consenso global sobre la necesidad de promover el desarrollo de tecnologías que disminuyan la emisión de gases de efecto invernadero, como el $\mathrm{CO}_{2}$, han impulsado la producción de productos petroquímicos a partir de materias primas basadas en fuentes renovables.

El reciclado es una opción pero no es una alternativa muy efectiva para todos los plásticos, en cambio, los biopolímeros suponen una solución desde el origen del problema ya que la gran mayoría de ellos su tiempo de biodegradación es mucho menor a la de un plástico común.

Los biopolímeros, que en su mayor parte proceden de recursos renovables por lo que se convierten en una interesante alternativa para la industria de los plásticos. (Ortiz Hernández. (2013)). Estos bioplásticos pueden procesarse mediante las mismas tecnologías que los materiales termoplásticos convencionales, tales como extrusión, inyección o soplado. Así, los polímeros basados en recursos renovables o biodegradables están generando un creciente interés, tanto en la sociedad en general como en la industria de los plásticos. (Valero Valdivieso et al. (2013))

Las membranas tienen varias aplicaciones desde el uso alimenticio como recubrimiento de comida como frutas $\mathrm{o}$ alimentos perecederos pueden incluir polisacáridos, compuestos de naturaleza proteica, lipídica o por una mezcla de los mismos, la última permite aprovechas las propiedades de cada compuesto y la sinergia entre ellos. (Quintero et al 2010) Dependiendo de las sustancias que los forman, los recubrimientos presentan ciertas propiedades mecánicas y de barrera al $\mathrm{H}_{2} \mathrm{O}, \mathrm{O}_{2}$ y $\mathrm{CO}_{2 \text {-. }}$ Así como en el área de la salud como recubrimientos de heridas en la piel.
El nopal (Opuntia ficus indica L. Millar) es una planta de la familia de las cactáceas que presenta características adaptativas en su anatomía, morfología y fisiología, las que le permiten sobrevivir, crecer y reproducirse en ambientes áridos y semiáridos (Galvez et al. (2017)). El mucílago de nopal en general (Opuntia spp.), obtenido de cladodios, es una sustancia hidrocoloidal, heteropolisacárida (con residuos de arabinosa, galactosa, ramnosa y xilosa como azúcares neutros); sus estructuras moleculares son polielectricas y altamente ramificadas y con peso molecular en el orden de los millones de Da (3 × 106 Da). (Madjdoub, H et al. (2001))

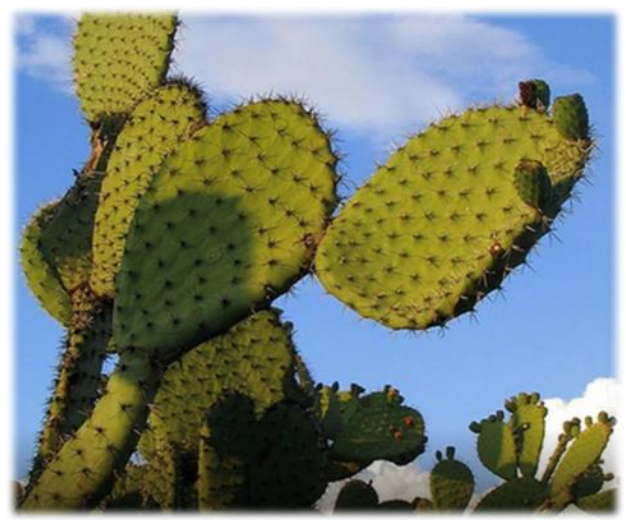

Figura 1 Nopal silvestre un.org

El objetivo del presente trabajo es elaborar y caracterizar membranas bioplásticas derivadas del Nopal (Opuntia ficus-indica) y conocer así sus propiedades físicas y químicas para posibles aplicaciones.

\section{Metodología}

La investigación se realizó en ciudad Obregón Sonora en las instalaciones del Instituto Tecnológico de Sonora, Campus Nainari en los laboratorios de Química LV 500, Laboratorio de Investigación de Ingeniería Química y Alimentos (LIIQA) y laboratorio de Ingeniería Civil LV 800.

Para la elaboración de la membrana bioplástica a base de Nopal (Opuntia ficusindica) a base de nopal, se efectuó un diseño experimental: Nopal - plastificante de origen vegetal creando membranas de tamaño estándar con apoyo de una malla, mostrado en la tabla1 donde se muestra la composición fraccional de cada componente. 


\begin{tabular}{|l|r|r|}
\hline Orden Corrida & Plastificante (P) & Nopal \\
\hline M1 & 0.317 & 0.367 \\
\hline M2 & 0.367 & 0.317 \\
\hline M3 & 0.300 & 0.400 \\
\hline M4 & 0.300 & 0.300 \\
\hline M5 & 0.333 & 0.333 \\
\hline M6 & 0.317 & 0.317 \\
\hline M7 & 0.350 & 0.350 \\
\hline M8 & 0.400 & 0.300 \\
\hline M9 & 0.300 & 0.350 \\
\hline M10 & 0.350 & 0.300 \\
\hline
\end{tabular}

Tabla 1 Composición experimental membranas

Se procedió a realizar las mezclas y someterlas a altas temperaturas hasta llegar a su secado. Texto redactado en Times New Roman No.12, espacio sencillo.

En la caracterización de las propiedades físicas de las membranas se realizaron las siguientes pruebas:

\section{Grosor}

Para determinar el grosor de las membranas se realizó la medición de las muestras de biopolímero, a través de un vernier starret. Para cada muestra, se tomaron 3 puntos distintos y se efectuó un promedio.

\section{Solubilidad}

Parámetro físico que nos permite establecer cuantitativamente, la capacidad de las membranas de disolverse en agua.

Para determinar la solubilidad de las membranas se procedió de la siguiente manera:

a. Para cada una de las muestras de membranas obtenidas, se obtuvieron 2 gramos de la muestra.

b. En un recipiente, previamente identificado, con el tipo de muestra a contener, volumen de agua y fecha de inicio, se agregaron los 2 gramos de muestra en $30 \mathrm{ml}$ de agua.

c. Se dejó la muestra de la membrana en la cantidad de agua indicada, agitándose cada día. El tiempo total en que se realizó la muestra, fue de 5 días.

d. Concluido el periodo de prueba, se obtuvo la cantidad de membrana disuelta en el agua.

\section{Permeabilidad}

Característica física de las membranas para analizar y establecer, la capacidad de retención o transferencia de agua hacia el exterior. Para determinar la permeabilidad de las membranas se procedió de la siguiente manera:

a. Se recortó la membrana del tamaño de la superficie a emplear como tapa (medio aislante).

b. Se identificaron los envases con la fecha y tipo de muestra de membrana a emplear.

c. Se procedió a medir las temperaturas de bulbo húmedo y seco.

d. A cada recipiente, se agregó la misma cantidad de agua, a fin de verificar la pérdida de agua para cada una de las muestras.

e. Se pesaron los envases con la cantidad de agua, envase y masa de la membrana, para monitorear la perdida de agua en cada día de medición.

f. Se taparon adecuadamente los envases que contienen el agua, con las diferentes muestras de membranas.

g. Se depositaron en un desecador.

h. La duración de la prueba, fue de 5 días, registrando en cada día: temperaturas de bulbo seco, húmedo y pesos.

i. Concluido el experimento, se procedió al cálculo de los valores de permeabilidad.

Prueba de gota. Indica de acuerdo con el ángulo de contacto, que tan hidrofóbico o hidrofílico es un biopolímero. Para determinar la prueba de gota de ambos biopolímeros se procedió de la siguiente manera:

Se determinó el índice de gota por medio del equipo OAD (Óptica Analysis Drop), el cual indica de acuerdo con el ángulo de contacto, que tan hidrofóbico o hidrofílico es el biopolímero.

Espectroscopia de infrarrojo. Identifica los grupos funcionales presentes en las membranas bioplásticas a base de Nopal. Para determinar esta característica de las membranas se necesitó la ayuda un espectrofotómetro el cual nos sirve para identificar los grupos funcionales presentes en el biopolímero. 


\section{Resultados}

Se elaboraron 10 membranas bioplásticas a base de Nopal con dimensiones de 40 X 50 X $0.2 \mathrm{Cm}$ mismas que para hacerle las pruebas se adaptaron en su tamaño.

Para cada uno de los biopolímeros se efectuaron seis pruebas diferentes las cuales son: grosor, solubilidad, permeabilidad, prueba de gota y espectroscopia de infrarrojo.

\section{Grosor y resistencia a la ruptura}

Se realizó la medición de las muestras de las membranas bioplásticas a base de Nopal a través de un vernier starret. Para cada muestra, se tomaron 3 puntos distintos. Se efectuó un promedio siendo este de $0.2 \mathrm{~cm}$. (MEC. Rogelio G. Garza Rivera, 2017)

En el grafico 1 se observa que la resistencia del material a la ruptura es constante hasta el límite proporcional a los 900 gramos. Posterior a esta medición, se observa que la resistencia del material se reduce significativamente, lo que ocasiona la ruptura de este

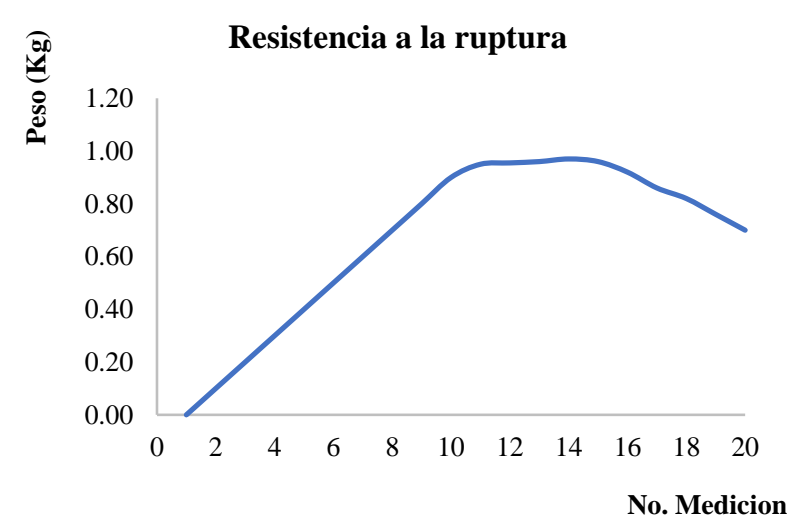

Gráfico 1 Resistencia a la ruptura de membranas bioplásticas a base de Nopal

\section{Solubilidad}

En esta prueba los resultados obtenidos se muestran en las siguientes tablas y gráficos:

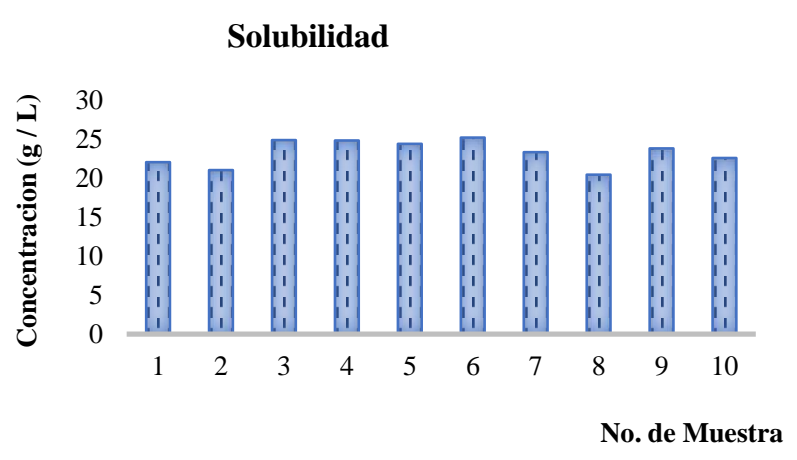

Gráfico 2 Solubilidad de membranas bioplásticas a base de Nopal

En el gráfica 2 se aprecia que la muestra 6 , es la que presenta mayor solubilidad (25.26 $\mathrm{g} / \mathrm{L})$, seguida de la muestra 3 (24.93 g/L). La muestra que presento menor solubilidad fue la muestra $8(20.48 \mathrm{~g} / \mathrm{L})$.

\section{Permeabilidad}

En el grafico 2 se observa que la permeabilidad de la muestra 3, es mayor, en tanto que la Permeabilidad de las muestras 1 y 10 , son menores y parecidas. La permeabilidad de las demás muestras no tiene variabilidad significativa.

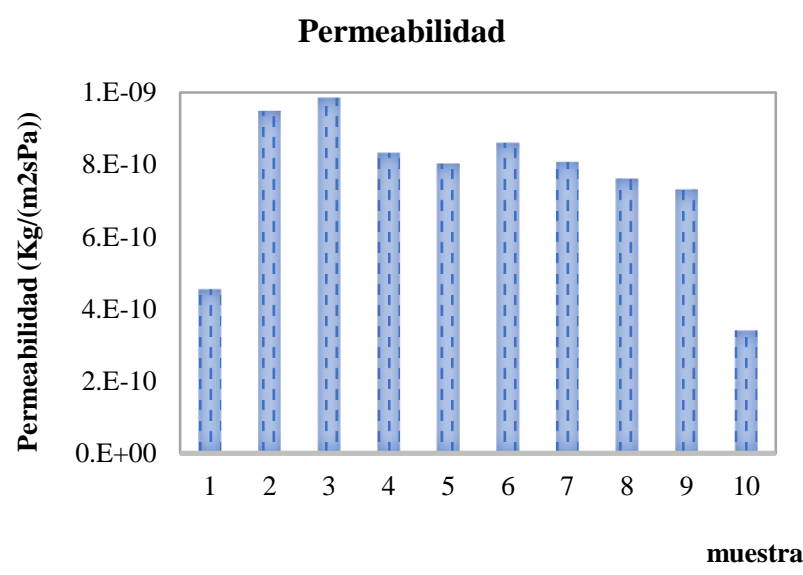

Gráfico 3 Permeabilidad de membranas bioplásticas a base de Nopal

Con esto se puede saber que las membranas a base de nopal no son tan permeables, es decir que nos permite menor paso de cantidad de agua por lo que si se quiere usar en un ambiente húmedo estos dos permiten el mínimo paso de humedad. 


\section{Prueba de gota}

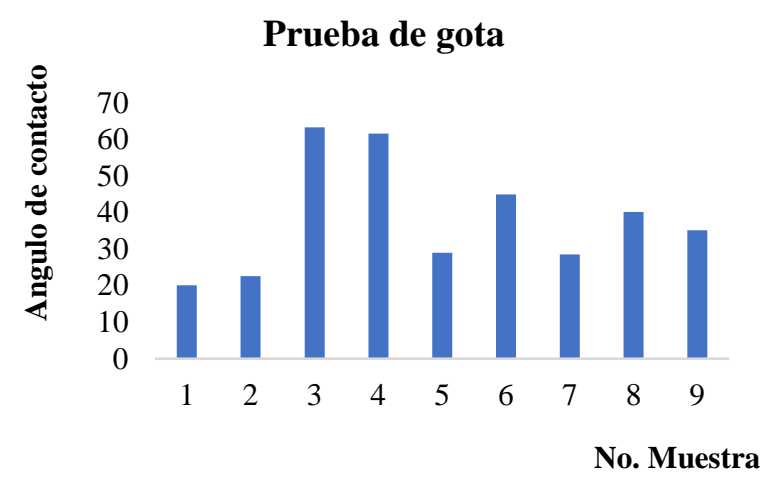

Gráfico 4 Angulo de gota de las membranas bioplásticas a base de Nopal

En la gráfica 4 se observa que las muestras que tienen una relación similar entre sí son las muestras 4, 6,8, 9 y 10 , el resto, tienen una variabilidad significativa.

Además, se observa que las muestras 3 y 5 , tienen un ángulo de contacto demasiado elevado, indicando son menos afines al agua. $\mathrm{La}$ muestra 7 , tiene un valor intermedio de solubilidad, mientras que las muestras 1,2 y 4 , tienen ángulos de contacto menores, lo que indica, son mucho más afines al agua.

\section{Espectroscopia de infrarrojo}

Se realizó un análisis de espectroscopia infrarrojo (EIR) para identificar los grupos funcionales presentes en las membranas bioplásticas a base de Nopal (Opuntia-ficus indica).

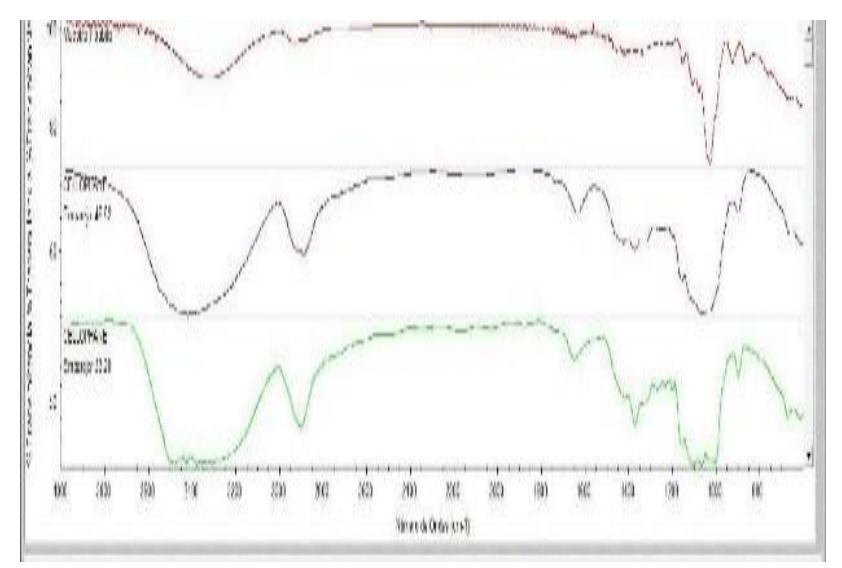

Figura 2 Espectroscopia de infrarrojo del Biopolímero de Nopal

Software OMNIC

En la figura 2 se observa la presencia del grupo carbonilo $(\mathrm{C}=\mathrm{O})$, alcohol $(\mathrm{R}-\mathrm{OH})$, y composición similar a la celulosa y grupo $\mathrm{OH}$ y Carbonilo siendo estos los más característicos.

\section{Conclusiones}

Con los resultados obtenidos se concluye que las membranas bioplásticas a base de nopal presentan características similares a las membranas convencionales, pero aun así se debe tomar en cuenta el uso se le desea dar ya que si se desea en algún medio húmedo o donde se requiera resistencia a la ruptura, pero esto varía de acuerdo a las cantidades de nopal y sábila, por lo tanto se concluye que las membranas bioplásticas a base de Nopal (Opuntia ficusindica), son similares a las membranas de plásticos sintéticos, siendo una alternativa viable para sustituirlos, contribuyendo a una mejor calidad de vida al ser amigable al cuidado del medio ambiente.

\section{Referencias}

Gálvez Chan Rosario Alicia, Silva Encinas Gabriel Omar, López Cuero Iván, Álvarez Sánchez Jesús y Paredes Gálvez Patricia Alejandra. (2017). Obtención y caracterización de una biopelícula a base de nopal (opuntia ficus-indica). En química e ingeniería verde para la sustentabilidad (473). Monterrey, México: Monterrey, México.

Jaimes N., Miguel A., Petróleo: historia y perspectivas geopolíticas. Aldea Mundo [en linea] 2012, 17 (Julio-Diciembre) : [Fecha de consulta: 27 de junio de 2018] Disponible en:<http://www.redalyc.org/articulo.oa?id=543 35426006> ISSN 1316-6727

Madjdoub, H; Roudesli, S.: Le Cerf, D.; Muller, G. \& Grisel, M. (2001). Prickly pear nopals pectin from Opuntia ficus indica. Physicochemical study in dilute and semidilute solutions. Carbohydrate polymers, 46: 69-78.

Ortiz Hernández María Laura. (2013). El impacto de los plásticos en el ambiente. 27 de mayo de 2013, de Centro de Investigación en Biotecnología de la Universidad Autónoma del Estado de Morelos (UAEM) Sitio web: http://www.jornada.unam.mx/2013/05/27/ecof.html

Quintero,C.J.P.,Pascual,F.V. y Muñoz, H.A.J.(2010).Peliculas y recubrimientos comestibles importancia y tendencias recientes en la cadena hortofrutícola.Revista Tumbaga, 5,93-118. 